\title{
CONDICIONES OCEANOGRÁFICAS Y COMPOSICIÓN DEL MESOZOOPLANCTON EN LA ZONA OCEÁNICA DEL PACIFICO COLOMBIANO, DURANTE SEPTIEMBRE-OCTUBRE 2004
}

\section{OCEANOGRAPHIC CONDITIONS AND MESOZOOPLANKTON COMPOSTION IN THE OCEANC ZONE OF COLOMBIAN PACIFIC, DURING SEPTEMBER-OCTOBER 2004}

\author{
Magnolia Murcia Riaño¹, Alan Giraldo López ${ }^{1,2}$ \\ ${ }^{1}$ Grupo de Investigación en Ecología Animal, \\ Departamento de Biología, Facultad de Ciencias, Universidad del Valle \\ A.A.25360. Cali - Colombia. Telefax: (57) (2) 3393243 \\ oceanografia@univalle.edu.co \\ ${ }^{2}$ Grupo de Estudios Oceanográficos y del Fenómeno de El Niño, \\ Área de Oceanografía Operacional, Centro Control Contaminación del Pacífico (DIMAR-CCCP) \\ Vía El Morro, Capitanía de Puerto de Tumaco, San Andrés de Tumaco, Nariño - Colombia. \\ oceanografia@univalle.edu.co
}

Murcia, M. y A. Giraldo. 2007. Condiciones oceanográficas y composición del mesozooplancton en la zona oceánica del Pacífico colombiano, durante septiembre-octubre 2004. Boletín Científico CCCP, (14): 83-94.

\section{Recibido en julio de 2007; aceptado en diciembre de 2007}

\section{RESUMEN}

Se estudió la composición del mesozooplancton y la variación espacial de las condiciones fisicoquímicas de la zona oceánica del Pacífico colombiano durante septiembre-octubre de 2004, a bordo del buque oceanográfico ARC Providencia. Se siguió un plan operativo de 21 estaciones de muestreo oceanográfico, de las cuales se realizaron arrastres de zooplancton en doce de ellas. El mesozooplancton se colectó mediante arrastres oblicuos con una red bongo desde $200 \mathrm{~m}$ de profundidad hasta la superficie. Adicionalmente, en cada estación de muestreo se registró la variación vertical de la temperatura (T) y salinidad (S) utilizando una sonda multiparamétrica CTD Seabird-19. Se realizaron muestreos discretos de agua a profundidad estándar $(0,10,20,30,50,75,100$ y 150m) utilizando una botella Niskin, con el propósito de cuantificar la concentración de oxígeno disuelto (OD) y la concentración de Clorofila-a (Chlo-a). Se definió la profundidad media de la capa de influencia friccional o capa de mezcla (CM) en la zona de estudio en $31.20 \pm 5.2 \mathrm{~m}$, siendo las condiciones oceanográficas promedio de $\mathrm{TCM}=26.8 \pm 0.3{ }^{\circ} \mathrm{C}, \mathrm{SCM}=32.9 \pm 0.4$, $\mathrm{OD}=4.57 \pm 0.1 \mathrm{ml} \mathrm{l}^{-1}$ y Clo-a $\mathrm{CM}=0.50 \pm 0.1 \mathrm{mg}$ Chlo-a m$~^{-3}$. Se identificaron 25 grupos taxonómicos, representando los copépodos el $71 \%$ del total de organismos, seguido por chaetognatos (6\%), sifonóforos $(6 \%)$, apendicularias (5\%), y ostrácodos y foraminíferos (2\%). La abundancia del mesozooplancton estuvo entre 79458 y 19962 Ind $100 \mathrm{~m}^{-3}$, mientras

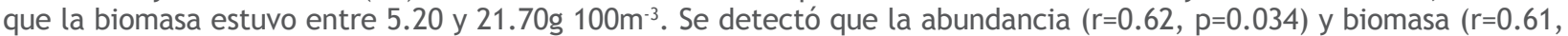
$\mathrm{p}=0.034$ ) de organismos mesozooplanctóncos estuvieron directamente correlacionadas con la concentración de clorofilaa en el estrato superior de la columna de agua $(0-50 \mathrm{~m})$. En este mismo sentido se estableció que la biomasa $(r=0.65$, $\mathrm{p}=0.023$ ) se correlacionó significativamente con la concentración de clorofila-a en la capa de influencia friccional del océano Pacífico colombiano.

PALABRAS CLAVE: mesozoopláncton, estructura comunitaria, biomasa, parámetros fisicoquímicos, Pacífico colombiano, ecología pelágica.

Taxonomic composition of mesozooplancton and spatial variability of physical - chemical and biological conditions in the oceanic zone of the Colombian Pacific Ocean were studied during September-October 2004 on board of B.O. ARC Providencia. 21 oceanographic stations were sampled, and only in 12 of them zooplankton haulages were carried out. Zooplankton was collected by oblique haulages of Bongo Net, from $200 \mathrm{~m}$ to surface. Vertical variation of temperature (T) and Salinity (S) was recorded with CTD Seabird ${ }^{-1}$. Samplings of water from standard depths $(0,10,20$, $30,50,75,100$ and $150 \mathrm{~m}$ ) were carried out using a Niskin bottle in order to quantify the concentration of dissolved oxygen (OD) and the concentration of Chlorophyll-a (Chlo-a). The depth of frictional influence layer or mixed layer $(\mathrm{ML})$ in the study zone was defined at $31.20 \pm 5.2 \mathrm{~m}$, being the mean oceanographic conditions of water column, $\mathrm{TCM}=26.8 \pm 0.3{ }^{\circ} \mathrm{C}, \mathrm{SCM}=32.9 \pm 0.4, \mathrm{OD}=4.57 \pm 0.1 \mathrm{ml} \mathrm{l}^{-1}$ and Clo-a $\mathrm{CM}=0.50 \pm 0.1 \mathrm{mg}$ Chlo-a m ${ }^{-3} .25$ taxonomic groups of zooplankton were identified, copépods representing $71 \%$ of the total of organisms, followed by Chaetognaths (6\%), Siphonóphors (6\%), Apendicularians (5\%), and Ostrácods and Foraminífera (2\%). The abundance of the mesozooplankton

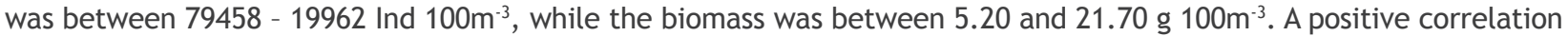
between abundance $(r=0.62, p=0.034)$ and biomass $(r=0.61, p=0.034)$ of mesozooplanktonic organisms with chlo-a concentration in the surface layer of water column $(0-50 \mathrm{~m})$ was detected. Moreover, the zooplankton biomass $(r=0.65, p=0.023)$ was significantly correlated with the concentration of chlo-a in the frictional influence layer of the Colombian Pacific Ocean.

KEY WORDS: mesozooplankton, community structure, biomass, Oceanography, Colombian Pacific, pelagic ecology, Oceanic waters, Eastern Tropical Pacific. 


\section{INTRODUCCIÓN}

El plancton ha sido definido por varios autores como el conjunto de organismos, tanto vegetales (fitoplancton) como animales (zooplancton), adultos y larvarios, que viven en las aguas dulces o marinas, ya sea flotando o dotados de escasos elementos de locomoción (Cifuentes et ál., 2000, Parsons et ál., 1984). Tanto el fitoplancton como el zooplancton son fuertemente influenciados por el conjunto de factores ambientales oceanográficos, en especial por la temperatura y la salinidad; parámetros que pueden llegar a condicionar la presencia o ausencia de una especie en una localidad determinada (Gasca et ál., 1996), aunque la posición espacial de estos organismos en el ambiente marino se encontrará modulada, principalmente, por los patrones de circulación locales (Mann y Lazier, 1996; Mullin, 1993), encontrándose una tendencia general a la agregación en zonas de frentes de discontinuidad térmicos (ver bordes de pluma de surgencia) o halinos (ver borde de pluma estuarina) (Shank, 1995; Gasca et ál., 1996). Además, cada especie responde de manera particular a los diferentes forzantes oceanográficos, lo que se refleja en un patrón de distribución biogeográfica discontinuo (Pérez-Ruzafa et ál., 2005)

Consecuentemente, no se puede considerar al zooplancton como un conjunto homogéneo de organismos ya que su distribución espacial no sólo cambiará en el espacio y el tiempo, debido a la influencia de forzantes físico-químicos, sino que también responde al efecto de algunos procesos biológicos, como la disponibilidad de alimento, predación y procesos de migración vertical, los cuales pueden llegar a condicionar la presencia o ausencia de estos organismos en una localidad (Seda y Deveter, 2000; Beisner, 2001; Avois-Jacquet et ál,. 2002)

De acuerdo con Parsons et ál. (1984), los organismos zooplanctónicos pueden ser clasificados de acuerdo con su tamaño en picoplancton (tamaño $<5 \mu \mathrm{m}$ ), microplancton (tamaño entre 20 y $200 \mu \mathrm{m}$ ) y mesoplancton (tamaño $>200 \mu \mathrm{m}$ ), siendo importantes para el sistema pelágico por su función como eslabón directo entre los productores primarios (fitoplancton) y consumidores superiores como peces, crustáceos y moluscos, que generalmente son de importancia comercial; de tal manera que se convierten en elementos estructurales de la productividad marina y de las redes tróficas pelágicas (González, 1988; Cifuentes et ál., 2000; Huskin et ál., 2001)

En el Pacífico colombiano diversos proyectos de investigación sobre zooplancton han sido desarrollados, aunque la mayoría de estas iniciativas se han enfocado hacia un grupo en particular (Escarria, 2006) o en ambientes costeros someros (Noriega, 1999; Patiño, 1999). Sin embargo, se destaca el trabajo realizado por Giraldo y Gutiérrez (2007), quienes evaluaron la composición del zooplancton superficial durante septiembre de 2003, registrando 26 grupos taxonómicos, una abundancia relativa entre 7900 y 105000 ind $100 \mathrm{~m}^{-3}$ y dominancia de copépodos (74\%) y quetognatos (8\%); y el trabajo de Giraldo y Martínez (2007), quienes evaluaron la composición del zooplancton en la corriente Colombia, durante febrero 2006, y su relación con los parámetros oceanográficos locales, registrando 28 grupos taxonómicos, una abundancia relativa entre 42200 y 1786700 ind $100 \mathrm{~m}^{-3}$, y la dominancia de salpas y goliólidos (49\%), y copépodos (31\%)

En términos generales, comprender el comportamiento de las poblaciones zooplanctónicas es relevante debido a que son el elemento que da inicio a las redes alimentarias en los océanos. Por lo tanto, el propósito de esta investigación fue caracterizar la estructura de la comunidad zooplanctónica en la zona oceánica de la cuenca del Pacífico colombiano (CPC) durante septiembreoctubre de 2004, evaluando el grado de asociación con los parámetros físicos, químicos y biológicos en la zona de estudio.

\section{ÁREA DE ESTUDIO}

La CPC se ubica geográficamente entre los paralelos $01^{\circ} 30^{\prime}$ hasta los $7^{\circ} 10^{\prime}$ de latitud Norte y entre los $77^{\circ} 40^{\prime}$ y $82^{\circ} 00^{\prime}$ de longitud Oeste. Sus límites geográficos son: las aguas del golfo de Panamá, al Norte; el litoral occidental de Colombia, integrado por los departamentos de Chocó, Valle del Cauca, Cauca y Nariño, al Este; las aguas de la costa ecuatoriana y la cordillera submarina de Carnegie, al Sur; y el océano Pacífico, las aguas territoriales de Panamá (30km) y la cordillera de Cocos, al Oeste. Las características geomorfológicas más importantes son isla Gorgona y la isla de Malpelo. El oleaje oceánico predominante en la CPC, al igual que el viento y la 


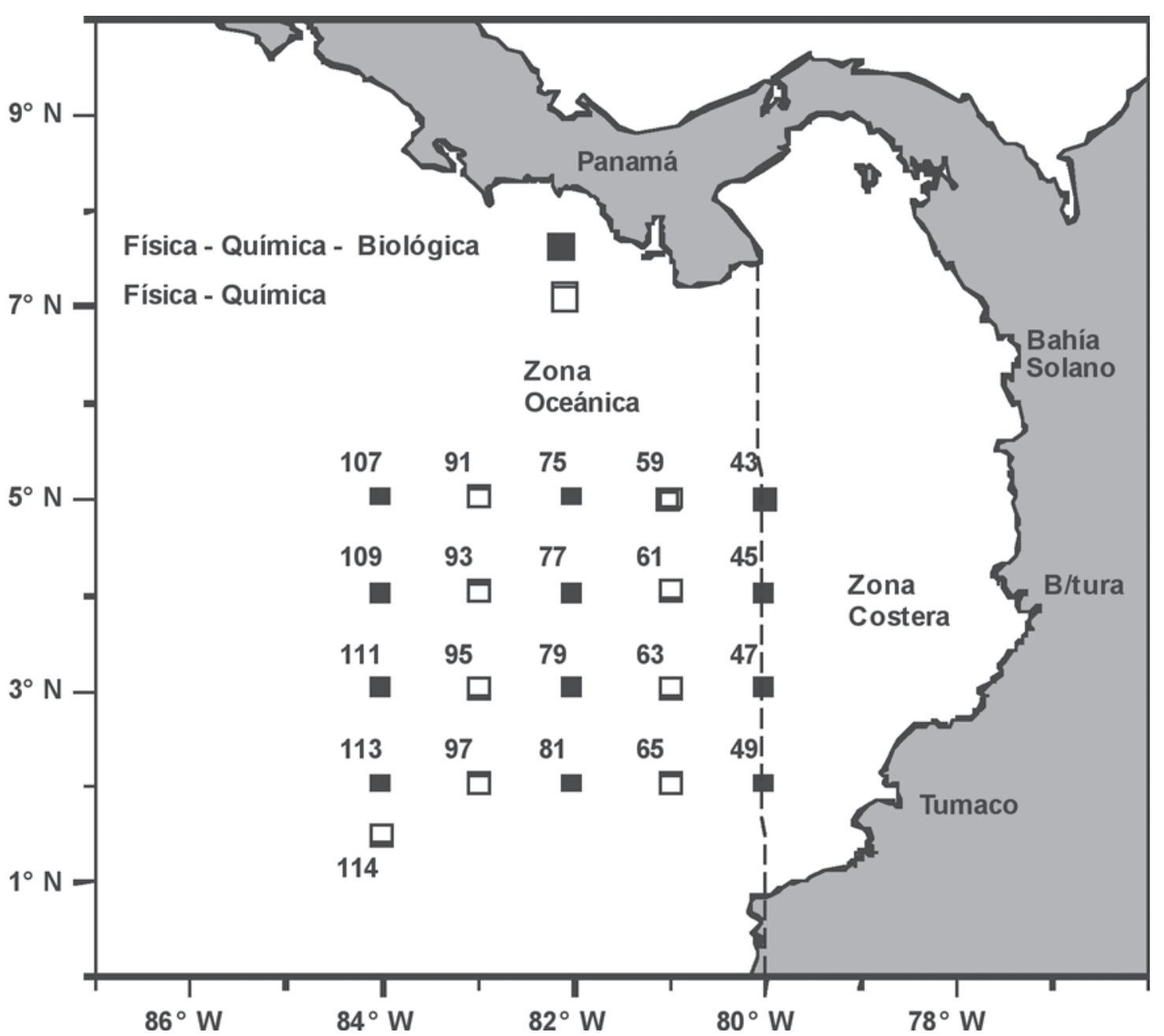

Figura 1. Zona de estudio y ubicación geográfica de las estaciones (físicas, químicas y biológicas) en la CPC, durante el crucero oceanográfico Pacífico XXXIX - ERFEN XXXVII a bordo del buque oceanográfico ARC Providencia.

corriente provienen del suroeste (Prahl et ál., 1990; Tejada et ál., 2003). Esta delimitación geográfica incluye a la CPC dentro de la franja conocida como la ensenada de Panamá (Panama Bight), la cual se extiende por las costas de Panamá, Colombia y Ecuador hasta la longitud $81^{\circ} \mathrm{W}$ (Fig. 1)

\section{METODOLOGÍA}

Las muestras fueron colectadas durante el crucero oceanográfico PACíFICO XXXIX - ERFEN XXXVII, a bordo del buque de la Armada Nacional ARC Providencia, entre septiembre y octubre de 2004 (Fig. 1). Para el desarrollo de este trabajo se consideró la zona oceánica en el área de estudio, como aquella región localizada más allá de los $80^{\circ} \mathrm{W}$. Se siguió un plan operativo de 21 estaciones de muestreo oceanográfico, realizando en doce de ellas arrastres oblicuos de zooplancton desde $200 \mathrm{~m}$ de profundidad hasta la superficie, con una red bongo de 300 y $500 \mu \mathrm{m}$, provista de un flujómetro digital para cuantificar el volumen de agua filtrada. Para el desarrollo de esta investigación se utilizó la muestra de zooplancton del colector de $300 \mu \mathrm{m}$, la cual fue preservada en frascos blancos de $500 \mathrm{ml}$ con formalina buferizada al $10 \%$ para el recuento taxonómico en el Laboratorio de Ecología Animal del Departamento de Biología de la Universidad del Valle.

Se realizó el registro de la abundancia de individuos estandarizada por el volumen de agua filtrado (Ind $100 \mathrm{~m}^{-3}$ ), considerando los grandes grupos 
taxonómicos funcionales a partir del análisis directo de las muestras mediante un estereoscopio NIKON (C-PS) con fuente de luz fría. Para la identificación y clasificación taxonómica se utilizaron las guías de Newell y Newell (1963) y Smith (1977). Se cuantificó el peso húmedo como indicador indirecto de la biomasa zooplanctónica, estandarizado por el volumen de agua filtrado (gr $\left.100 \mathrm{~m}^{-3}\right)$. Este método consistió en pasar toda la muestra por un tamiz de $60 \mu \mathrm{m}$, extraer el exceso de agua con papel absorbente y transferir la muestra a un pedazo de papel aluminio previamente pesado, registrando el peso con una balanza analítica $( \pm 0.00001 \mathrm{~g})$ Denver Instrument Company $\left(\mathrm{AA}^{-1} 60\right)$

Se determinó la concentración de Clorofilaa profundidades estándar $(0,10,20,30,50$, 75,100 y $150 \mathrm{~m}$ ), como un indicador de la biomasa fitoplanctónica mediante el método de extracción con acetona (90\%) y cuantificación por espectofotometría. Se estimó la concentración de $\mathrm{OD}$, mediante el método de titulación (Winkler), y se registraron las condiciones de temperatura y salinidad en la columna de agua mediante el uso de una sonda multiparamétrica perfiladora CTD Seabird19. Todos los análisis químicos fueron realizados en el Laboratorio de Química Marina del Centro Control Contaminación del Pacífico (CCCP)

Considerando que el patrón de distribución espacial del zooplancton no es aleatorio, se utilizó un análisis no paramétrico de correlación múltiple para establecer el grado de asociación entre las variables biológicas (abundancia y biomasa zooplanctónica) y las variables oceanográficas (temperatura, salinidad, OD y clorofila-a) en la zona de estudio. Todos los análisis estadísticos se realizaron utilizando el programa Statistica versión 5.1 y Minitab versión 14.1. Con el propósito de establecer los patrones de distribución espacial de las variables biológicas y oceanográficas se realizó un análisis gráfico descriptivo utilizando la rutina de interpolación Krigging del programa SURFER $®$ versión 8.0.

\section{RESULTADOS}

Se identificaron un total de 25 grupos mesozooplanctónicos (Tabla I), destacándose por su abundancia relativa componentes del holopláncton como copépodos que constituyeron un $71 \%$ del total de la comunidad de organismos, seguido por quetognatos $(6 \%)$, sifonóforos $(6 \%)$, apendicularias (5\%) y en menor proporción se encontraron ostrácodos y foraminíferos (2\%) (Fig. 2). De los 25 grupos taxonómicos identificados 17 estuvieron presentes en todas las estaciones de muestreo, por lo que se pueden clasificar como grupos constantes [(porcentaje de ocurrencia >50\% (Mujica y Rojas 1985)], estos grupos fueron: copépodos, quetognatos, salpas y doliolos, sifonóforos, medusas, apendicularias, anfípodos, gasterópodos, ostrácodos, eufáusidos (adultos y larvas), poliquetos, pterópodos, foraminíferos, larvas de peces, mysidaceos, squilla, cladóceros, zoeas y bivalvos. En este mismo sentido, las megalopas, cefalópodos y mytilus fueron clasificados como grupos accesorios (porcentaje de ocurrencia entre 25 y $50 \%$ ), mientras que isópodos y urocordados fueron clasificados como grupos accidentales (porcentaje de ocurrencia entre 0 y $25 \%$ )

La abundancia total fue altamente variable con registros de 79458 Ind $100 \mathrm{~m}^{3}$ en el sector norte (E75) y 49632 Ind $100 \mathrm{~m}^{3}$ en el sector sur (E49) (Fig. 3). La biomasa mesozooplanctónica estuvo

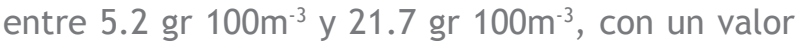

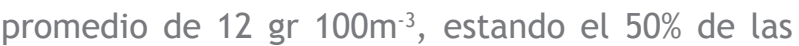
estaciones por debajo del promedio; sin embargo, es importante destacar que los mayores valores de biomasa se asociaron a estaciones ubicadas sobre los $82^{\circ} \mathrm{W}$. Al examinar la relación entre la biomasa y la abundancia del mesozoopláncton no se encontró una correlación significativa $(r=0.55, p=0.068)$

La temperatura promedio de la capa de influencia friccional o zona de mezcla, considerada desde la superficie hasta la primera inflexión del perfil de temperatura, fue de $26.8^{\circ} \pm 0.3^{\circ} \mathrm{C}$, con un rango entre $27.2{ }^{\circ} \mathrm{C}$ (E59) y $26.3{ }^{\circ} \mathrm{C}$ (E114) (Fig. 4A). Es importante mencionar el desarrollo de un núcleo de baja temperatura $\left(19^{\circ} \mathrm{C}\right)$ a $30 \mathrm{~m}$ de profundidad en la zona oeste del área de estudio $\left(3^{\circ} \mathrm{N}\right.$ a $\left.83^{\circ} \mathrm{W}\right)$. La salinidad promedio de la capa de mezcla $(0 \mathrm{a}$ $30 \mathrm{~m}$ ) fue de $32.9 \pm 0.4$, con un valor máximo de 33.4 (E113) y un valor mínimo de 31.9 (E43) (Fig. 4B). Se detectó una fuerte estratificación vertical en toda la zona de estudio, delimitándose un estrato superficial en la columna de agua ( 0 a $30 \mathrm{~m}$ ) asociada, probablemente, a la capa de influencia friccional (Fig. 4C). La concentración de OD en la capa de influencia friccional estuvo entre $4.71 \mathrm{ml} \mathrm{L}^{-1}$ (E 43) a $4.48 \mathrm{ml} \mathrm{L}^{-1}$ (E 79), mientras que la concentración de Clorofila-a estuvo entre $0.74 \mathrm{mg} \mathrm{Clo-a} \mathrm{m}^{-3}$ (E75) y $0.36 \mathrm{mg}$ Clo-a m ${ }^{-3}$ (E113) 


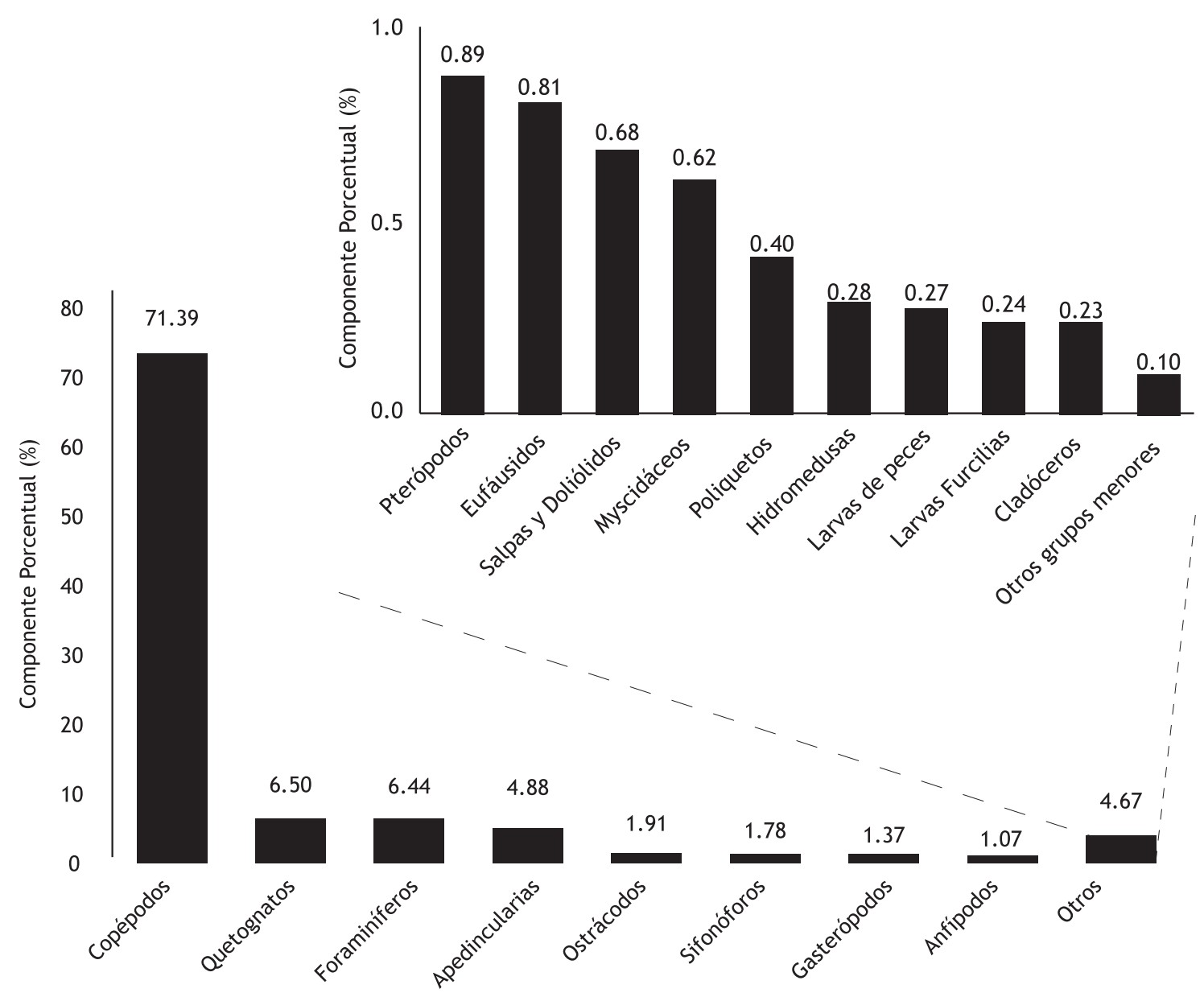

Figura 2. Composición porcentual del zooplancton presente en la zona oceánica del océano Pacífico colombiano durante el crucero oceanográfico PACÍFICO XXXIX - ERFEN XXXVII.

Se detectó una asociación significativa entre la abundancia del mesozooplancton y la concentración de clorofila-a en el estrato de 0 a $50 \mathrm{~m}(r=0.62$, $\mathrm{p}=0.034$ ). En este mismo sentido se encontró que la biomasa mesozooplanctónica se relacionó con la clorofila-a de la capa de mezcla (0 a $30 \mathrm{~m})$, la clorofila-a de 0 a 50m ( $r=0.65, p=0.023 ; r=0.61$, $p=0.034)$ y el OD en la columna de agua ( $r=0.73$, $p=0.008$ ). No se detectaron relaciones significativas entre la abundancia y biomasa del mesozooplancton y la temperatura, salinidad o densidad de la columna de agua.

\section{DISCUSIÓN}

Diversos estudios sugieren que los parámetros ambientales juegan un rol importante en la distribución de las comunidades planctónicas, especialmente sobre las fluctuaciones latitudinales de abundancia. En este sentido tanto los procesos físicos como los biológicos generan un sistema natural multi-escalar sobre la cual se presenta la variabilidad del zooplancton (Haury et ál., 1978; Legendre et ál., 1986; Avois-Jacquet et ál., 2002)

En esta investigación se detectó un núcleo de alta densidad en la zona oceánica $\left(82^{\circ} \mathrm{W}\right.$ a $\left.5^{\circ} \mathrm{N}\right)$ para la abundancia zooplanctónica, y menores valores hacia el resto de la cuenca con algunos núcleos en el transecto Tumaco $\left(2^{\circ} \mathrm{N}\right)$, observándose una aparente heterogeneidad en toda la cuenca, no obstante una de las principales características de las comunidades zooplanctónicas es que presentan una alta heterogeneidad espacial (Seda y Devetter, 2000; Beisner 2001), en consecuencia el patrón de distribución estará modulado por factores ambientales complejos que operan a diferentes 
Tabla I. Abundancia (Ind $100 \mathrm{~m}^{-3}$ ) de los grupos zooplanctónicos colectados durante el crucero oceanográfico PACÍFICO XXXIX - ERFEN XXXVII.

\begin{tabular}{|c|c|c|c|c|c|c|c|c|c|c|c|c|c|}
\hline TAXA & 43 & 45 & 47 & 49 & 75 & 77 & 79 & 81 & 107 & 109 & 111 & 113 & $\begin{array}{l}\text { IND/ } \\
\text { TAXA }\end{array}$ \\
\hline Copépodos & 21093 & 32970 & 31350 & 27861 & 69528 & 10470 & 22348 & 33993 & 27239 & 27554 & 19806 & 35692 & 359904 \\
\hline Chaetognatos & 3738 & 2689 & 2654 & 6542 & 2351 & 3597 & 1664 & 3445 & 964 & 1586 & 1248 & 2276 & 32753 \\
\hline $\begin{array}{l}\text { salpas } \\
\text { y doliolos }\end{array}$ & 546 & 282 & 138 & 249 & 314 & 341 & 130 & 274 & 466 & 151 & 85 & 452 & 3428 \\
\hline Sifonóforos & 456 & 988 & 823 & 706 & 583 & 405 & 526 & 1451 & 1211 & 400 & 608 & 792 & 8948 \\
\hline Medusa & 543 & 10 & 3 & 51 & 11 & 520 & 4 & 26 & 77 & 105 & 8 & 34 & 1393 \\
\hline Apendicularia & 1732 & 1192 & 3466 & 3080 & 1311 & 179 & 1274 & 2282 & 3410 & 2347 & 624 & 3694 & 24590 \\
\hline Anfípodo & 106 & 589 & 401 & 195 & 724 & 1118 & 581 & 373 & 170 & 182 & 706 & 228 & 5373 \\
\hline Megalopa & 0 & 3 & 3 & 12 & 0 & 0 & 0 & 0 & 4 & 0 & 7 & 6 & 35 \\
\hline Gasterópodo & 882 & 1041 & 639 & 414 & 414 & 382 & 590 & 696 & 73 & 375 & 812 & 582 & 6899 \\
\hline Ostrácodo & 1034 & 698 & 800 & 4075 & 395 & 341 & 410 & 326 & 255 & 488 & 358 & 472 & 9654 \\
\hline Zoea & 9 & 31 & 23 & 8 & 0 & 7 & 4 & 3 & 0 & 7 & 0 & 3 & 95 \\
\hline Eufáusido & 385 & 522 & 200 & 113 & 313 & 500 & 421 & 656 & 142 & 130 & 587 & 97 & 4065 \\
\hline Furcilia & 348 & 225 & 65 & 66 & 47 & 7 & 105 & 89 & 69 & 98 & 21 & 91 & 1231 \\
\hline Poliqueto & 199 & 353 & 302 & 137 & 325 & 54 & 70 & 96 & 138 & 112 & 78 & 178 & 2041 \\
\hline Pterópodo & 612 & 703 & 433 & 82 & 631 & 274 & 495 & 214 & 190 & 109 & 535 & 206 & 4484 \\
\hline Bivalvo & 34 & 84 & 3 & 62 & 22 & 0 & 27 & 3 & 0 & 7 & 18 & 13 & 274 \\
\hline Foraminífero & 463 & 656 & 2385 & 4945 & 1890 & 1189 & 3528 & 2654 & 1924 & 6269 & 2394 & 4157 & 32453 \\
\hline Larvas pez & 208 & 143 & 79 & 12 & 101 & 169 & 122 & 33 & 110 & 109 & 123 & 141 & 1349 \\
\hline Cefalópodo & 0 & 0 & 0 & 12 & 0 & 0 & 6 & 10 & 0 & 0 & 3 & 6 & 37 \\
\hline Mysidáceo & 295 & 340 & 138 & 105 & 467 & 388 & 489 & 280 & 251 & 109 & 143 & 95 & 3101 \\
\hline Squilla & 43 & 20 & 29 & 78 & 17 & 20 & 8 & 86 & 12 & 74 & 66 & 47 & 502 \\
\hline Cladócero & 71 & 7 & 0 & 20 & 14 & 0 & 0 & 0 & 4 & 972 & 0 & 88 & 1176 \\
\hline Isópodo & 3 & 0 & 0 & 0 & 0 & 0 & 0 & 0 & 0 & 0 & 0 & 0 & 3 \\
\hline Urocordado & 3 & 0 & 0 & 0 & 0 & 0 & 0 & 0 & 0 & 0 & 1 & 2 & 6 \\
\hline Mytilus & 0 & 0 & 0 & 4 & 0 & 0 & 12 & 26 & 0 & 0 & 21 & 278 & 341 \\
\hline IND/EST & 32803 & 43545 & 43936 & 48828 & 79458 & 19962 & 32815 & 47016 & 36708 & 41184 & 28251 & 49632 & 504137 \\
\hline
\end{tabular}

escalas; tales como el efecto de las fuerzas físicas advectivas causado por la influencia de los giros o 'eddies', régimen de vientos, gradientes de turbidez, disponibilidad de alimento y condiciones tróficas (predación y competencia)
Aunque los análisis no evidenciaron una fuerte relación con los parámetros físicos, se sugiere que la alta densidad de organismos registrada hacia la zona norte puede estar favorecida por los valores intermedios de temperatura $\left(26.8^{\circ} \mathrm{C}\right)$ y salinidad, 


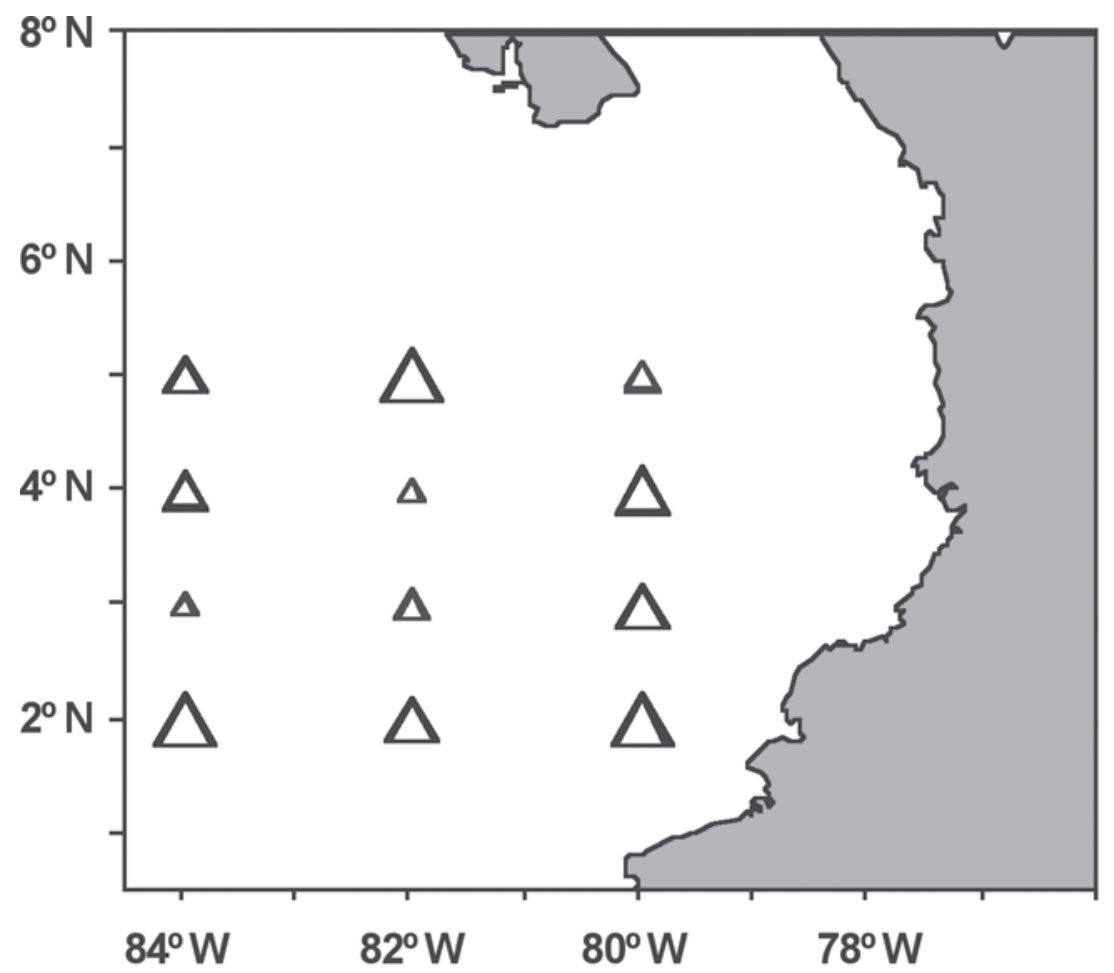

Abundancia (Ind 100 $\mathrm{m}^{-3}$ )
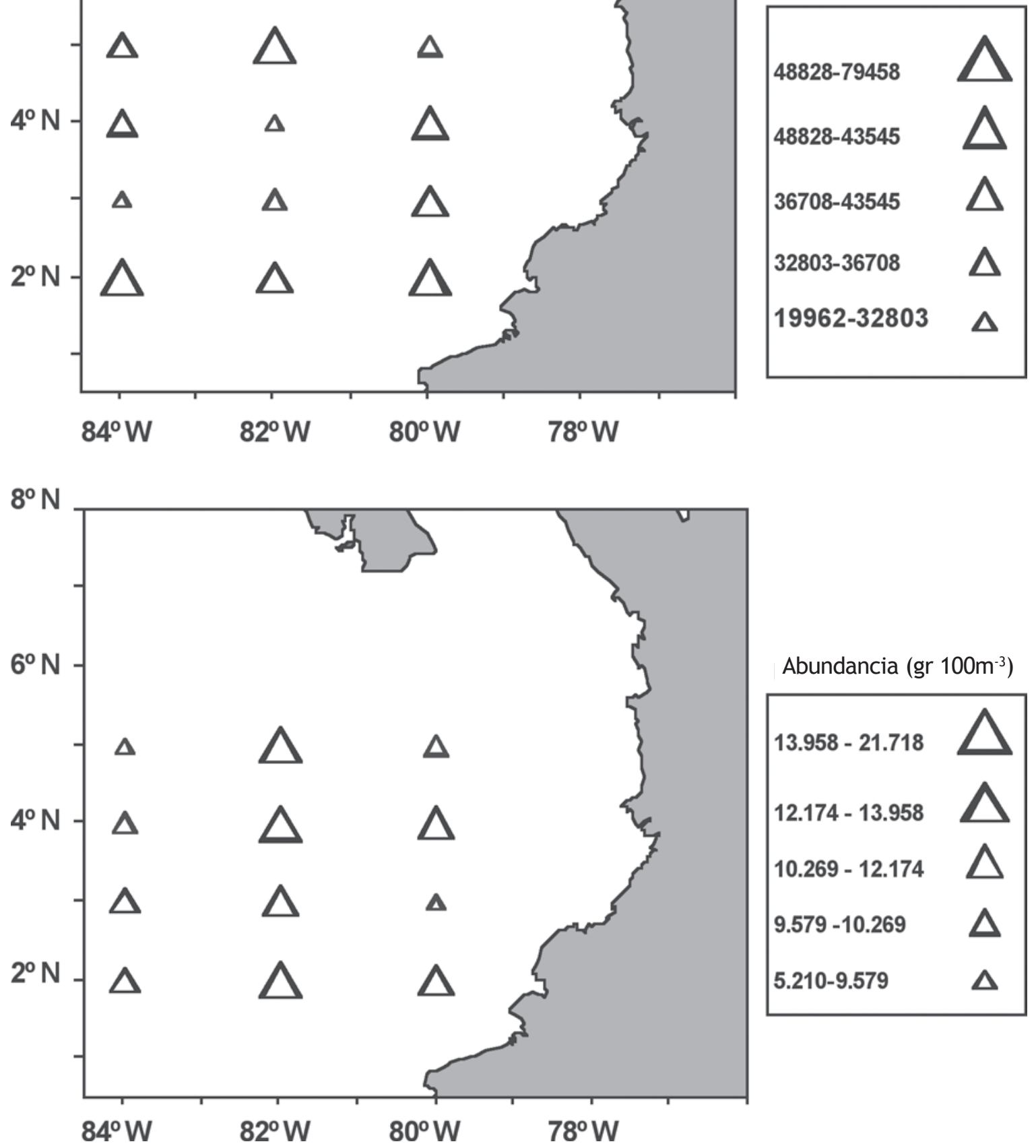

Abundancia (gr 100m³)

$\mid \begin{array}{ll}13.958-21.718 & \Delta \\ 12.174-13.958 & \Delta \\ 10.269-12.174 & \Delta \\ 9.579-10.269 & \Delta \\ 5.210-9.579 & \Delta\end{array}$

Figura 3. Distribución espacial de la abundancia (Ind $100 \mathrm{~m}^{-3}$ ) y la biomasa $\left(\mathrm{gr} \mathrm{m}^{-3}\right)$ en la zona oceánica del Pacífico colombiano durante el crucero oceanográfico PACÍFICO XXXIX - ERFEN XXXVII. 

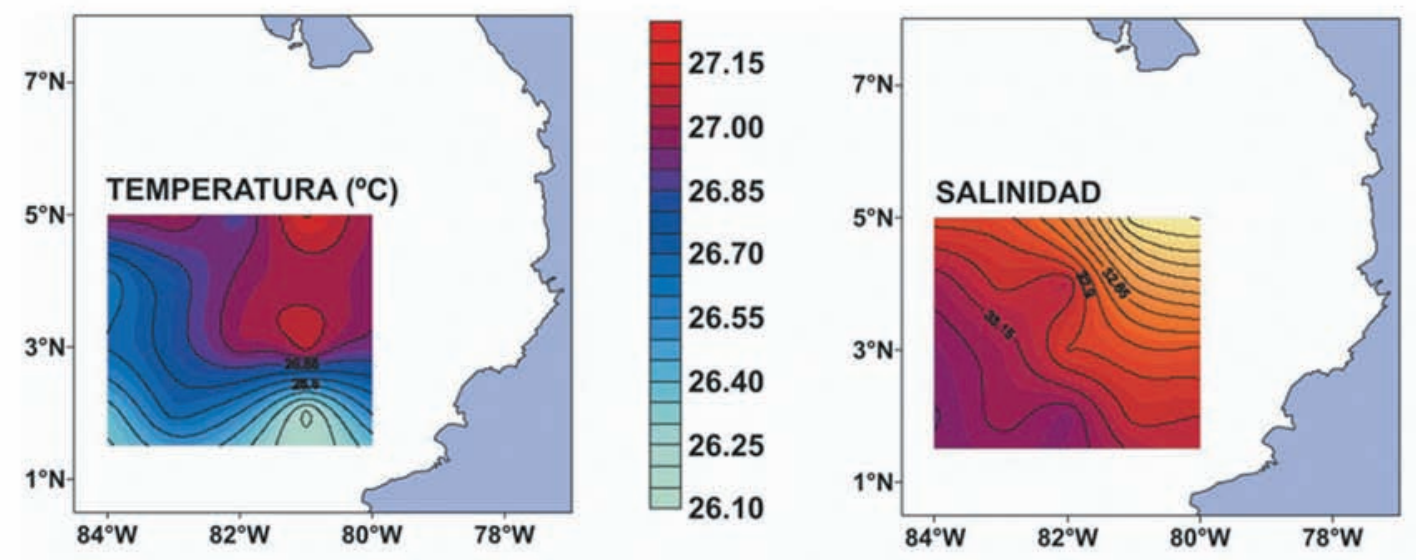

33.30

33.10

32.90

32.70

32.50

32.30

32.10
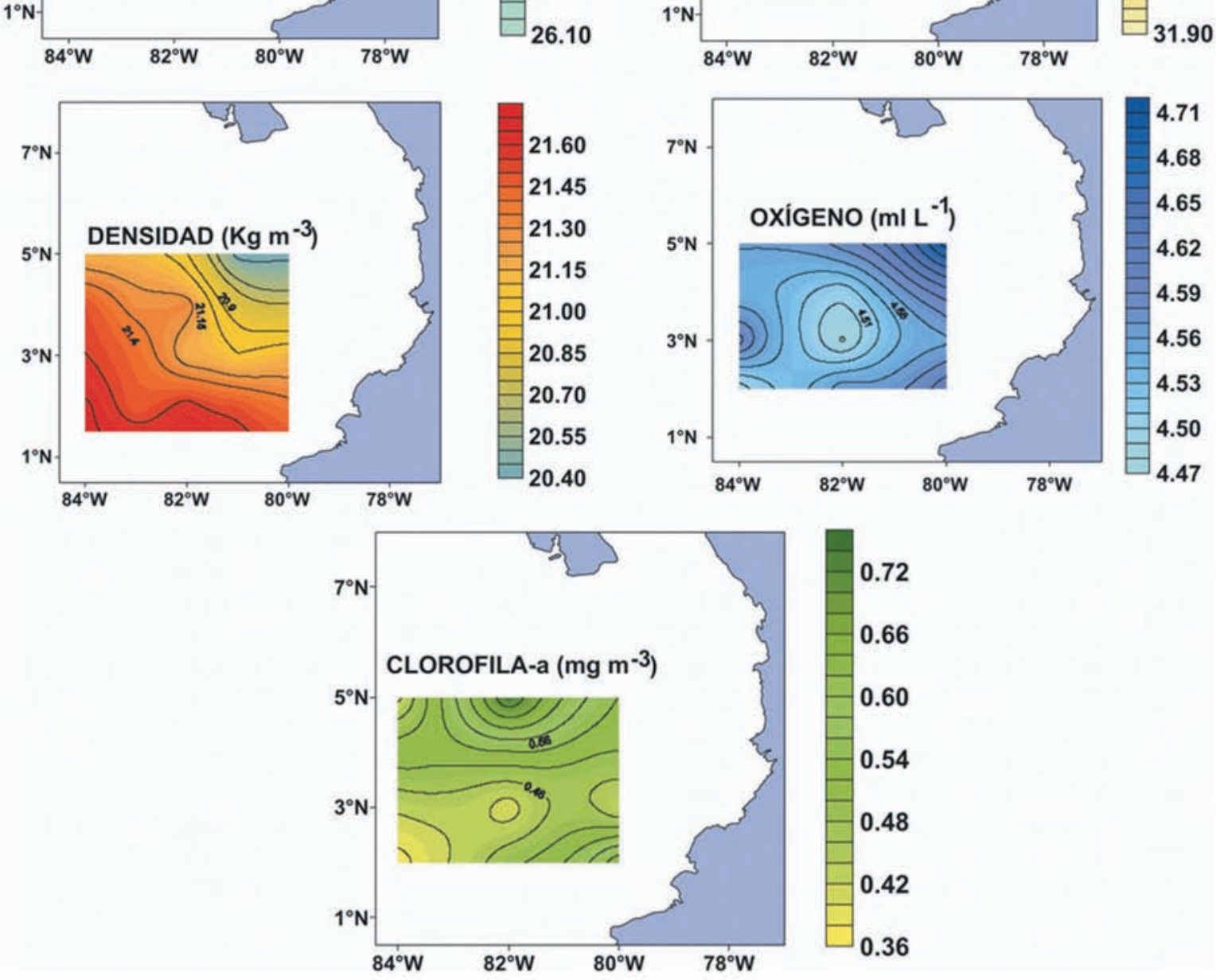

Figura 4. Distribución espacial de los parámetros físicos [temperatura $\left({ }^{\circ} \mathrm{C}\right)$, salinidad, densidad $\left(\mathrm{Kg} \mathrm{m}^{-3}\right)$ ], químicos [OD $\left(\mathrm{ml} \mathrm{L}^{-1}\right)$ ] y biológicos [Clorofila-a $\left.\mathrm{mg} \mathrm{m}^{-3}\right)$ ] en la zona oceánica del Pacífico colombiano durante el crucero oceanográfico PACÍFICO XXXIX - ERFEN XXXVII. 
lo que concuerda con los resultados reportados por García (2001), quien observó una tendencia a encontrar una mayor densidad de organismos zooplanctónicos en sectores con valores intermedios de temperatura superficial para la época de muestreo, como en el sector noreste de la cuenca. Sin embargo, en el extremo sudoeste de la cuenca se detectó un núcleo de alta abundancia, asociado a temperatura baja, lo que sugiere la presencia de una entrada de agua 'fría' proveniente del sur.

Las aguas del Pacífico colombiano están encerradas en un sistema mayor, conocido como Panama Bight. Éste posee algunas características climáticas y oceanográficas particulares, debido a que está sometido a variaciones estacionales de temperatura y salinidad causadas por la migración, a lo largo del año, de la zona de convergencia intertropical (ZCIT) y los vientos Alisios del norte y del sur, los cuales se desplazan desde los trópicos hacia el Ecuador geográfico, arrastrando consigo un alto porcentaje del agua evaporada de la superficie del mar (Forsbergh, 1969)

El patrón de circulación superficial de esta región tropical del Pacífico es complejo y responde normalmente al sistema dominante de los vientos planetarios, determinados por el desplazamiento de la ZCIT. Las aguas superficiales se caracterizan por ser relativamente cálidas $\left(25\right.$ a $\left.26{ }^{\circ} \mathrm{C}\right)$ y de baja salinidad (33.5 frente a la costa colombiana) (Prahl et ál., 1990). Según Braun et ál., (2005), al momento de realizar el muestreo oceanográfico (septiembre-octubre de 2004), las condiciones atmosféricas globales observadas a partir de junio de 2004 sugerían un comportamiento anómalo, contrastando con las zonas costeras del Pacífico oriental, tal como se observó en la zona norte del Pacífico colombiano que presentó precipitaciones entre ligeras a moderadas.

Los resultados de esta investigación son consistentes con lo registrado por Gutiérrez (2004) y Giraldo y Gutiérrez (2007) en cuanto a la estructura, abundancia y relación entre el mesozoopláncton superficial y las condiciones oceanográficas en la cuenca del Pacífico colombiano. Estos autores, reportaron una alta homogeneidad para la abundancia del mesozoopláncton superficial en toda la cuenca y la ausencia de asociación entre la abundancia y la temperatura o salinidad. Sin embargo, es importante destacar que la estratificación detectada en la columna de agua durante la presente investigación puede tener fuertes implicaciones para el zooplancton. En este sentido, la estratificación vertical potenciaría la variabilidad de las condiciones físicas químicas y biológicas entre diferentes estratos de la columna de agua, incluyendo el alimento (Caffrey et ál., 1994; Edmunds et ál., 1995, 1997; Baylosis et ál., 1997); la temperatura, la cual afecta la demanda metabólica (McLaren, 1974; Caffrey et ál., 1994; Edmunds et ál., 1995, 1997; Baylosis et ál., 1997); la turbulencia, la cual afecta la alimentación (Saiz et ál., 1992); los predadores y competidores (Ambler et ál., 1985; Orsi y Mecum, 1986), y la incidencia de luz y corrientes (Morgan et ál., 1997)

Es de esperar que el principal aporte a la biomasa zooplanctónica en la zona de estudio sea por el grupo más abundante, en este caso los copépodos y organismos con poco contenido de líquidos en su cuerpo (eufáusidos, larvas de decápodos, etcétera). De acuerdo con Boltovskoy (1981), la biomasa generalmente brinda una mejor información sobre la proporción de la comunidad que está ligada al tamaño de los organismos; sin embargo, en esta investigación la biomasa de la comunidad estuvo modulada principalmente por el número de individuos, más que por su tamaño. Es importante destacar que durante la estimación de la biomasa del zooplancton no se consideró el aporte de los huevos o larvas de peces presentes en las muestras.

Contrario a lo encontrado por García (2001), en algunas estaciones de muestreo se estableció una relación inversa abundancia-biomasa, encontrando sitios con baja abundancia y altos estimados de biomasa seca. Bajo esas condiciones es probable que la biomasa esté modulada por el tamaño de los organismos más que por la abundancia de éstos. Por ejemplo, García (2001) encontró en la estación 75 una baja abundancia; sin embargo, esta estación oceánica registró una alta presencia de megalopas de cangrejo que contribuyeron para el estimado de peso seco de la comunidad en esta estación. Por el contrario, durante la presente investigación se registró en una estación costera una alta abundancia de túnicas gelatinosas de thaliaceos de gran tamaño que no incidieron en el porcentaje en peso obtenido.

En conclusión, el mesozoopláncton en la zona oceánica del Pacífico colombiano durante septiembre-octubre de 2004 estuvo dominado por 
copépodos (herbívoros), destacándose además por su abundancia los quetognatos (carnívoros). De acuerdo con los resultados de esta investigación es de esperarse que la estructura y composición del mesozooplancton en el Pacífico colombiano dependa, principalmente, del campo de corrientes y masas de agua presentes en la zona de estudio, aunque la concentración de clorofila-a sería el parámetro biológico que más estaría influenciando la abundancia y biomasa de la comunidad mesozooplanctónica.

\section{AGRADECIMENTOS}

Los autores del presente estudio expresan agradecimientos a la Dirección General Marítima (DIMAR) y al Centro de Control de Contaminación del Pacífico (CCCP), por permitir la participación en la campaña oceanográfica PACÍFICO XXXIX - ERFEN XXXVII y el apoyo brindado durante la fase de campo.

Igualmente manifiestan gratitud a los departamentos de Biología (Laboratorio de Biología) y Física (Laboratorio de Física) de la Universidad del Valle, por facilitar espacio y equipos de laboratorio requeridos para el análisis cuantitativo de las muestras biológicas. El muestreo biológico se realizó en conjunto con B. Beltrán de la Unidad Administrativa Especial del Sistema de Parques Naturales Nacionales (UAESPNN)

Este trabajo fue financiado parcialmente por la Universidad del Valle (Vicerrectoría de Investigaciones, Facultad de Ciencias y Departamento de Biología) y la DIMAR-CCCP, en el marco del proyecto de investigación 'Producción primaria, biomasa fitoplanctónica y composición taxonómica del fitoplancton y zooplancton del Pacífico colombiano: una aproximación holística a la base del sistema pelágico - Cl 7662' a favor de A. Giraldo.

\section{LTTERATURA CTIADA}

Ambler, J . W. , J . E. Cloern y A. Hutchinson. 1985. Seasonal cycles of zooplankton from San Francisco Bay. Hydrobiology, (129): 177-197.

Avois-J acquet, C., P. Legender y M. Louis. 2002. Coastal tropical zooplankton: Patterns and processes over spatial scales. Submitted to Coral Reefs.
Baylosis, J. I., J. L. Edmunds, B. E. Cole y J. E. Cloern. 1997. Studies of the San Francisco Bay, California estuarine ecosystem: pilot regional monitoring results, 1996. U.S. Geol. Survey OpenFile Rep. 203 pp.

Beisner, B. E. 2001. Plankton community structure in fluctuating environments and the role of productivity. OIKOS, 95 (3):496-510.

Braun, M., J . Ortiz, E. Pinto, T. de la Cuadra, E. Zambrano, R. Flores, C. Grados, L. Pizarro y U. Munaylla. 2005. Comportamiento Meteorológico y Oceanográfico en el Pacífico Sudeste durante septiembre-octubre de 2004. Informe Final. VII Crucero Regional Conjunto de Investigación Oceanográfica en el Pacífico Sudeste. Guayaquil, Ecuador. 46 pp.

Boltovskoy, D. (ed.) 1981. Atlas del zooplancton del Atlántico Sudoccidental y métodos de trabajo con el zooplancton marino. Instituto Nacional de Investigación y Desarrollo Pesquero. Mar de la Plata, Argentina, 936 pp.

Caffrey, J. M., B. E. Cole, J . E. Cloern, J . R. Rudek, A. C. Tyler y A. D. J assby. 1994. Studies of the plankton and its environment in the San Francisco Bay estuary, California, regional monitoring results, 1993. U.S. Geol. Survey Open-File Rep. 411 pp.

Centro Control Contaminación del Pacífico. 2003. Compilación Oceanográfica de la Cuenca Pacífica Colombiana. (Ed.) DIMAR. San Andrés de Tumaco: CCCP, Serie Publicaciones Especiales Vol. 1, 109 $\mathrm{pp}$.

Cifuentes, J . L., P. Torres-García y M. Frías. 2000. El océano y sus recursos: Plancton. 2 ed. Fondo de Cultura Económica. México. 161pp.

Edmunds, J. L., B. E. Cole, J. E. Cloern, J.M. Caffrey y A.D. J assby. 1995. Studies of the San Francisco Bay, California, estuarine ecosystem. Pilot regional monitoring program results, 1994. U.S. Geol. Survey Open- File Rep. 436 pp.

Edmunds, J. L., B. E. Cole, J. E. Cloern y R. G. Dufford. 1997. Studies of the San Francisco Bay, California, estuarine ecosystem. Pilot regional 
monitoring program results, 1995. U.S. Geol. Survey Open-File Rep. 380 pp.

Escarria, E., B. S. Beltrán-León y A. Giraldo. 2006. Ictioplancton superficial de la cuenca del océano Pacífico colombiano (septiembre 2003). Invest. Mar., Valparaíso, 34 (2): 169-173.

Forsbergh, E. D. 1969. On the climatology, oceanography and fisheries of the Panama Bight. Bull. Iter.-am Trop Tuna Comm., 14 (2): 49-365.

Gasca, R., L. Segura y E. Suárez. 1996. El zooplancton marino. En: Gasca, R. \& E. Suárez (Eds). Introducción al estudio del zooplancton marino. ECOSUR-CONACYT. México, pp. 1-36.

Giraldo, A. y E. Gutiérrez. 2007. Composición taxonómica del zooplancton superficial en el Pacífico colombiano (septiembre 2003). Investig. mar., 35 (1): 117-122.

Giraldo, A. y T. Martínez. 2007. Respuesta local de la comunidad zooplanctónica del sistema pelágico del Pacífico colombiano a forzantes de gran escala. Informe técnico. Universidad del Valle, Facultad de Ciencias, Departamento de Biología, 23 pp.

González, A. 1988. Plancton de las aguas continentales. Secretaría General de la Organización de los Estados Americanos, Washington, D.C., $130 \mathrm{pp}$.

Gutiérrez, E. 2004. Estructura de la comunidad zooplanctónica del océano Pacífico colombiano y su relación con variables físicas, químicas y biológicas. Cali, 59 pp. Trabajo de grado (Biología Marina) Universidad del Valle.

Haury, L. R., J. S. McGowan y P. Wiebe. 1978. Patterns and Processes in the time-space scales of plankton distributions. In: Steele JH (ed) Spatial pattern in plankton communities. Plenum Press, New York, pp. 277-327.

Huskin, I., R. Anadon., G. Medina., R. N. Head y R. P. HARRIS. 2001. Mesozooplankton distribution and copepod grazing in the Subtropical Atlanctic near the Azores: Influence of mesoscale structures. Journal of Plankton Research, 23 (7): 671-691.
Legendre, L., S. Demers y D. Lefaivre. 1986. Biological production at marine ergoclines. In: Nihoul JC (ed) Marine interfaces ecohydrodynamics. Elsevier, Ámsterdam, pp 1-29.

Mann, K. H. y J . R. N. Lazier. 1996. Dynamics of marine ecosystems: biological-physical interactions in the oceans, $2^{\text {nd }}$ ed. Boston, Blackwell Science, 394 pp.

McLaren, I. A. 1974. Demographic strategy of vertical migration by a marine copepod. Am. Nat. (108): 91-101.

Morgan, C. A. , J . R. Cordell y C. A. Simenstad. 1997. Sink or swim? Copepod population maintenance in the Columbia River estuarine turbidity-maxima region. Mar. Biol. (129): 309- 317.

Mujica, A. y O. Rojas. 1985. Composición y abundancia del zooplancton del norte de Chile, Crucero MARCHILE XV-ERFEN VI, Diciembre de 1983. CIEN. Y TEC. DEL MAR. CONA, (9): 31-52.

Mullin, M. M. 1993. Webs and scales: physical and ecological processes in marine fish recruitment. Washington Sea Grant. University of Washignton Press. 135 pp.

Newell, G. E y R. C. Newell. 1963. Marine Plankton a practical guide. Hutchinson, London, USA. 207 pp.

Noriega, M. 1999. Evaluación de la productividad secundaria y su relación con parámetros fisicoquímicos en el modelo de la calidad de aguas para la Ensenada de Tumaco, Pacífico Colombiano. Informe final de ECOPETROL. Modelo de Calidad de las Aguas para la Ensenada de Tumaco y Plan de Seguimiento y Vigilancia de la contaminación Marina en el Pacífico colombiano Fase II. Centro de Control de Contaminación del Pacífico, San Andrés de Tumaco, 69 pp.

Orsi, J. J. y W. L. Mecum. 1986. Zooplankton distribution and abundance in the Sacramento-San Joaquin delta in relation to certain environmental factors, Estuaries 9 (4B): 326-339.

Patiño, J. 1999. Variación espacio-temporal del zooplancton en la ensenada de Tumaco, costa 
Pacífica colombiana. Periodo mayo-diciembre 1999. Convenio ECOPETROL- CCCP. Centro Control Contaminación del Pacífico. San Andrés de Tumaco. $25 \mathrm{pp}$.

Parsons, T. R., M. Takahashi y B. Margrave. 1984. Biological Oceanographic processes. $3^{\text {th }}$ ed. Pergamon Press, Great Britain, 330 pp.

Pérez-Ruzafa, A.,C. Marcos y J.J. Bacallado. 2005. Biodiversidad marina en archipiélagos e islas: patrones de riqueza específica y afinidades faunísticas. Universidad de la Rioja. España, (33): 455-476.

Prahl, H., J. R. Cantera y R. Contreras. 1990. Manglares y hombres del Pacífico colombiano. Editorial Presencia. Cali, Colombia, 184 pp.
Saiz, E., M. Alcaraz y G. A. Paffenhoffer. 1992. Effect of small scale turbulence on feeding rate and gross growth efficiency of three Acartia species. J. Plankton Res. (14): 1085-1097.

Shank, A. 1995. Mechanisms of cross-shelf dispersal of larval invertebrates and fish. En: McEDWARD. L. (Ed). Ecology of marine invertebrate larvae. CRC Press, Boca Ratón, Fl. Pp. 323 - 368.

Seda, J. y M. Devetter. 2000. Zooplankton community structure along a trophic grandient in a canyon-shaped dam reservoir. Journal of Plankton Research, 22 (10):1829-1840.

Smith, D. L. 1977. A guide for Marine Coastal Plankton and Marine Invertebrate Larvae. Kendall/ Hunt, California, USA. 161pp. 